\section{Synthesis of Proteins with Disulfide Bonds in $E$. coli Using Defined Culture Media}

BioTechniques 32:1238-1242 (June 2002)

Synthesis of eukaryotic proteins containing disulfide bonds in E. coli normally produces misfolded products because the low redox potential of the bacterial cytosol is not appropriated for the formation of the disulfide bonds of these polypeptides. Although in many cases those denatured proteins can be refolded to a native conformation, the yield of this process is usually very low and the product too heterogeneous for high-resolution structural studies. Several eukaryotic cell-based systems have been described as alternatives to the $E$. coli-based ones. Nevertheless, those systems are considerably more cumbersome and more expensive. Moreover, with eukaryotic systems, it is difficult to obtain a homogeneously labeled protein, either with seleno-methionine for crystallographic analyses or with $15 \mathrm{~N}$ and ${ }^{13} \mathrm{C}$ for $\mathrm{NMR}$. Therefore, the development of expression systems that allow the synthesis in native conformation of eukaryotic disulfide bondcontaining proteins in E. coli grown in defined media is still an attractive biotechnological goal. Several systems for synthesizing this type of protein in E. coli (in native conformation) have been delineated that have worked successfully in some cases. Such is the case of the E. coli strains in which the thioredoxin and glutaredoxin reduction pathways have been knocked out to raise the thiol-disulfide redox potential of the cytoplasm (2). Other approaches, such as the pIN-III-ompA vector-based systems (9), couple protein synthesis to its secretion to the more oxidizing environment of the periplasm, where the disulfide bond-forming and isomerizing enzymes are present. However, labeling requires the culture to be grown in minimal medium, a condition in which protein synthesis using pIN-III-ompA vectors becomes quite inefficient (C.F.-T., personal communication). Accordingly, polypeptide synthesis of medium- or large-scale quantities using ompA vec- tors usually requires culture media enriched with either casamino acids or yeast extracts $(5,11)$.

We observed that one of the products of the Ricinus communis $2 \mathrm{~S}$ seed storage protein, termed RicC3 (1), is toxic for $E$. coli when it accumulates in its cytoplasm, but not if added to the culture medium. This protein was used for the development of an E. coli strain able to secrete the products of the cloned genes into the periplasm when it is grown in minimal medium. RicC3 is a $14.4-\mathrm{kDa}$ polypeptide with two disulfide bonds.

In the first step, the RicC3 gene was inserted between the EcoRI-HindIII sites of pIN-III-ompA, creating the vector pINompA-RicC3, which was subsequently transformed into $E$. coli BL21. A significant accumulation of a polypeptide with the $M_{\mathrm{r}}$ of RicC3 was observed in the periplasm of the cells grown in LB, $60 \mathrm{~h}$ after protein synthesis induction with $1 \mathrm{mM}$ IPTG. We did not observe any apparent decrease in the viability of the culture because of the accumulation of $\mathrm{RicC} 3$ in the periplasm. In addition, this polypeptide was also detected in the culture medium, where actually most of the RicC3 protein accumulated. Figure 1A, lanes 1 and 2, illustrates that RicC 3 accumulated in the medium at considerably higher levels when the cells were grown in minimal medium supplemented with casamino acids than when they were grown in LB medium. The polypeptide could easily be isolated from the culture medium in analytical quantities by reverse-phase chromatography, and the sequence was unequivocally identified as RicC3 by Edman degradation. The cultures were not viable after induction of protein synthesis when the cells were grown in minimal medium, a condition in which the secretion of the proteins targeted to the periplasm by ompA vectors works deficiently (5).

In the second step, the cells harboring the pINompA-RicC3 vector $(1 \mu \mathrm{L}$ overnight culture) were plated on Petri dishes of minimal medium containing 1 $\mathrm{mM}$ IPTG and $200 \mu \mathrm{g} / \mathrm{mL}$ ampicillin. After incubation at $37^{\circ} \mathrm{C}$, an average of 12 colonies/plate were retrieved (a continuous cell lawn was instead obtained when the same amount of cells were plated in the presence of only the antibiotic). The cells from most of these colonies secrete into the culture medium equivalent levels of RicC3 when they are grown in minimal broth and in the same medium supplemented with casamino acids, respectively (exemplified in Figure 1A, lanes 3 and 4). These RicC3 levels are even higher than those produced by the parent cells grown in minimal medium supplemented with casamino acids (Figure 1A, lane 2). In a final step, a culture of one of these colonies was treated with sub-lethal doses of novobiocin to eliminate the pINompA-RicC3 plasmid (10). Isolates of these treated cells, retransformed with pINompA-RicC3 and grown in minimal medium, yielded comparable expression levels of RicC3 to those produced by cells from which they were directly derived (Figure 1A, lanes 4 and $5)$. The new cell strain has been designated BL21-SS. RicC3 can be easily purified, at a semi-preparative scale, from the supernatant of this strain, cultured in minimal medium, by a combination of ammonium sulfate precipitation, SPSepharose ${ }^{\circledR}$ (Amersham Biosciences, Uppsala, Sweden) and reverse-phase chromatographies with a final yield of approximately $12 \mathrm{mg} / \mathrm{L}$ culture. The circular dichroism (CD) spectrum of purified recombinant RicC3, synthesized using BL21-SS, is indistinguishable from that of the protein purified from seeds (Figure 1B). Those spectra appear typical of unfolded proteins when the disulfide bonds were reduced and the cysteines blocked by carboxymethylation (data not shown). Calculation of the $\alpha$-helical content of RicC3, from the $[\theta]_{222}$ of the spectra of Figure 1B (3), yielded a value (approximately $44 \%$ ) of the order of that shown by the 3-D structure of napin (55\%), a protein highly homologous to RicC3 (14).

In pIN-III-ompA vectors, the expression of proteins is controlled by a quite leaky promoter, which may represent a serious inconvenience in the case of proteins whose periplasmic accumulation is toxic. To overcome such a limitation, we developed a new expression plasmid that, in addition to targeting the cloned protein into the periplasm and the culture medium, maintains its expression under tight control. The new vector was also designed for the optional incorporation of a poly-histidine tag at the C-terminus of the cloned protein that can be 
removed from the purified polypeptide using the human rhinovirus $3 \mathrm{C}$ protease. This new expression vector was designated pRHO.

The pRHO vector (Figure 2) is com posed by the 4246-bp HindIII-NcoI fragment from pRAT-4 (12) plus two small fragments flanking the target gene that were constructed from synthetic oligonucleotides using the overlappingoligonucleotides polymerizing method
(6). The 74-bp fragment upstream of the target gene contains a sequence encoding the ompA signal peptide with the best codon usage for $E$. coli. Immediately following the coding sequence are two alternative cloning sites for the target gene, EcoRI and NaeI (Figure 2). NaeI is blunt-ended but has the advantage of allowing the desired protein to be cloned without addition of extra amino acids at the N-terminus. The

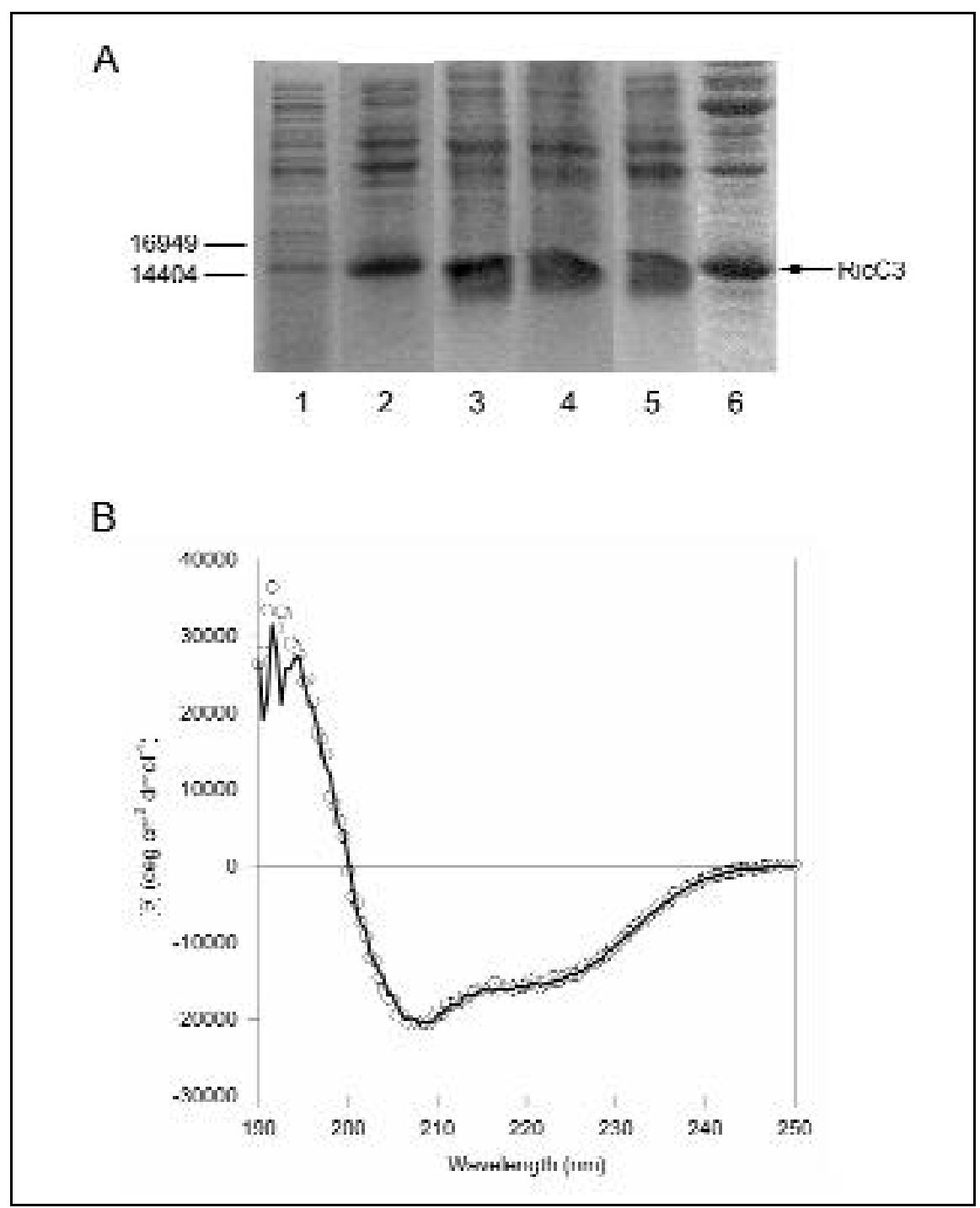

Figure 1. Expression of recombinant RicC3. Panel A compares the extracellular expression levels of RicC3 from pIN-III-ompA and pRHO in different culture media, monitored by SDS-PAGE. In the case of pIN-III-ompA (lanes 1-5), the cells were induced with $1 \mathrm{mM}$ IPTG when $A_{800}=0.3$, and, after $60 \mathrm{~h}$ growth at $37^{\circ} \mathrm{C}$, a $100-\mu \mathrm{L}$ sample of the supernatant of the culture (centrifuged at $15000 \times g$ for $15 \mathrm{~min}$ ) was run on a $16 \%$ polyacrylamide gel in tricine buffer and stained with Coomassie ${ }^{\circledR}$ Blue. RicC3 expression levels in the extracellular medium of E. coli strains BL21 (lanes 1-4), BL21-SS (lane 5), and BL21(DE3)-SS (lane 6). E. coli was grown in LB (lane 1), M9 + 0.4\% casamino acids (lanes 2 and 3), and M9 alone (lanes 4-6). The BL21(DE3)-SS strain containing pRHO-RicC3 (lane 6) was grown for $24 \mathrm{~h}$ after induction with $1 \mathrm{mM}$ IPTG at $\mathrm{A}_{000}=0.8$. Molecular weights are indicated on the left. Panel $\mathrm{B}$ shows the CD spectra of both purified recombinant RicC3 expressed from pIN-III-ompA in BL21-SS cells (black line) and RicC3 purified from $R$. communis (open circles).

112-bp fragment downstream of the target gene includes a sequence that encodes the rhinovirus $3 \mathrm{C}$ protease recognition site (3CRS) (4) and the poly-histidine tag (his6), followed by a set of translation terminators in all three reading frames (13). The his6 tag coding sequence (together with that of the 3CRS) can be omitted from the expressed protein by cloning its gene between the EcoRI/NaeI and StuI sites.

The pRHO vector was tested with positive results for the expression of RicC3 in an appropriate E. coli strain [BL21(DE3)] grown either in LB or minimal medium supplemented with casamino acids. As above, induction of protein expression in minimal medium cultures caused cell death, an effect that was again used to select a BL21(DE3) variant that efficiently secretes proteins into the culture medium under those conditions. This new BL21(DE3) mutant host, BL21(DE3)-SS, was capable of secreting high amounts of RicC3 from $\mathrm{pRHO}$ into the culture medium when grown in minimal broth (Figure 1A, lane 6). The levels of RicC3 obtained with the pRHO vector and the BL21(DE3)-SS strain were equivalent to those obtained with the other strategies reported here. Nevertheless, shorter times were required to reach those levels (60 versus $24 \mathrm{~h}$ ), which may help to minimize the potentially undesirable modifications that exposure to the culture medium may introduce in the expressed protein.

Proteins containing 2-7 disulfide bonds, such as the $\mathrm{N}$ - and C-terminal fragments of the insulin-like growth factor binding protein- 4 , have been successfully synthesized in native conformation with the help of the new vectors and strains reported here (C.F.-T., personal communication). Proteins with no cysteine residues were also effeciently produced, such as the C-terminal domain of pneumococcal autolysin $(7,8)$. In all cases, the final purification yield averaged $10 \mathrm{mg} / \mathrm{L}$ minimal medium culture. Several of these polypeptides are currently being used for high-resolution structural studies (NMR and X-ray diffraction) with promising results. The $\mathrm{N}$ - and $\mathrm{C}$ terminal fragments of the insulin-like growth factor binding protein-4 were expressed at very low yields in other existing hosts and vectors for expression and 


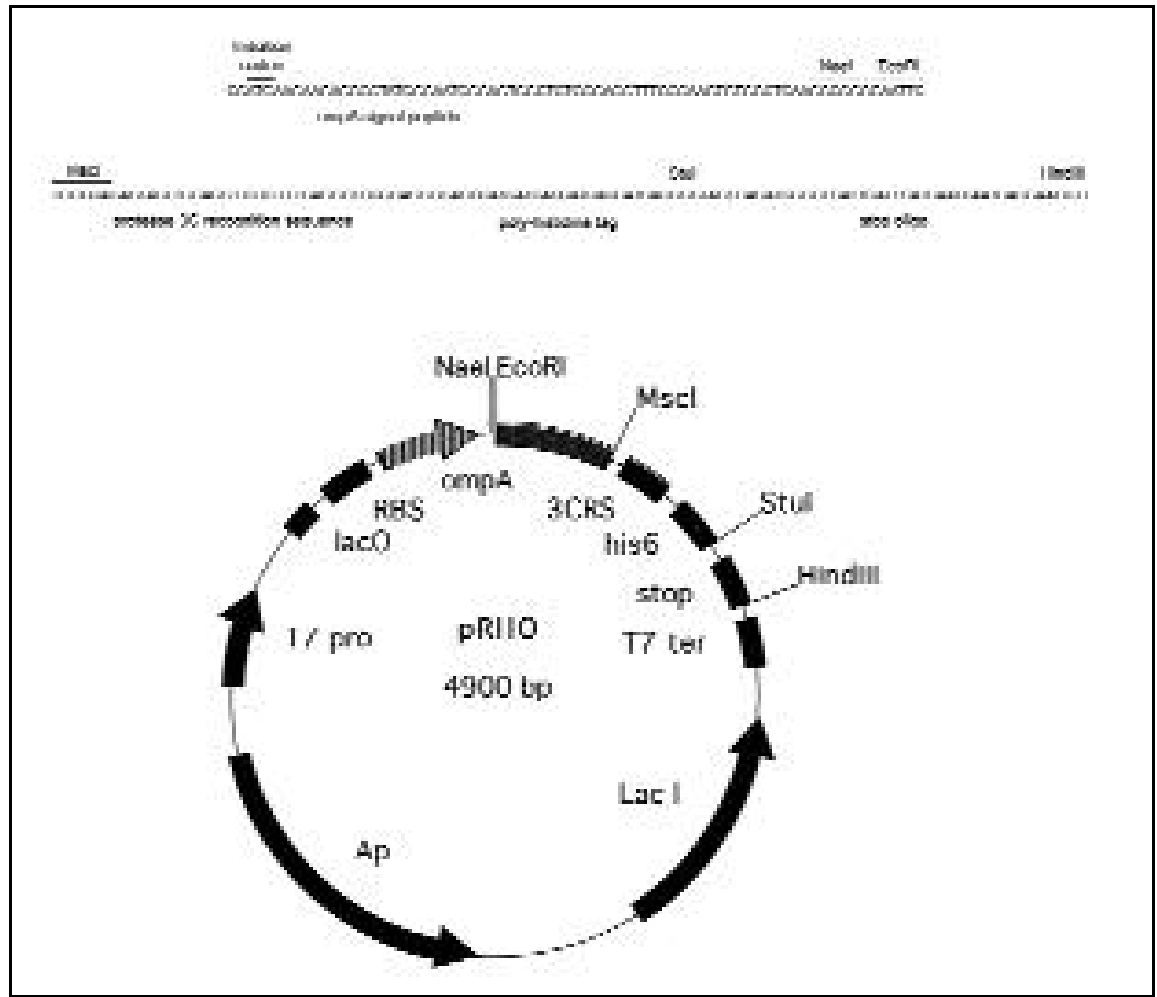

Figure 2. Schematic drawing of the pRHO vector. pRHO contains the T7lac promoter, the ribosomebinding site $(g 10 L)$ and transcription terminator $(T \phi)$ from pRAT4. The sequence of the newly synthesized fragments upstream and downstream of the cloning sites is shown above the vector. The drawing was created with MacPlasmap v. 1.82 (University of Utah, Salt Lake City, UT, USA).

refolding of disulfide-rich proteins referenced at the beginning this report (2).

In summary, we have developed a new system for high-level expression of heterologous proteins in their native conformations using cultures of $E$. coli in minimal medium. This system seems especially suitable for eukaryotic proteins containing disulfide bonds, which need to be labeled with ${ }^{15} \mathrm{~N}$ and ${ }^{13} \mathrm{C}$ for NMR studies. The strategy reported here could also be used to develop $E$. coli strains for labeling proteins with selenium, a desirable strategy for X-ray crystallography.

\section{REFERENCES}

1.Bashir, M.E., I. Hubatsch, H.P. Leinenbach, M. Zeppezauer, R.C. Panzani, and I.H. Hussein. 1998. Ric c 1 and Ric c 3, the allergenic $2 \mathrm{~S}$ albumin storage proteins of Ricinus communis: complete primary structures and phylogenetic relationships. Int. Arch. Allergy Immunol. 115:73-82.

2.Bessette, P.H., F. Åslund, J. Beckwith, and G. Georgiou. 1999. Efficient folding of proteins with multiple disulfide bonds in the Es- cherichia coli cytoplasm. Proc. Natl. Acad. Sci. USA 96:13703-13708.

3.Chen, Y.H., J.T. Yang, and H.M. Martínez. 1972. Determination of the secondary structures of proteins by circular dichroism and optical rotatory dispersion. Biochemistry 11:4120-4131.

4.Cordingley, M.G., P.L. Callahan, V.V. Sardana, V.M. Garsky, and R.J. Colonno. 1990. Substrate requirements of human rhinovirus 3C protease for peptide cleavage in vitro. J. Biol. Chem. 265:9062 -9065.

5.Denèfle, P., S. Kovarik, T. Ciora, N. Gosselet, J.C. Bénichou, M. Latta, F. Guinet, A. Ryter, and J.F. Mayaux. 1989. Heterologous protein export in Escherichia coli: influence of bacterial signal peptides on the export of human interleukin 1 $\beta$. Gene 85:499-510.

6.Di Donato, A., M. de Nigris, N. Russo, S. Di Biase, and G. D'Alessio. 1993. A method for synthesizing genes and cDNAs by the polymerase chain reaction. Anal. Biochem. 212:291-293.

7.Fernández-Tornero, C., R. López, E. García, G. Giménez-Gallego, and A. Romero. 2001. A novel solenoid fold in the cell wall anchoring domain of the pneumococcal virulence factor LytA. Nat. Struc. Biol. 8:1020-1024.

8.Fernández-Tornero, C., Á. Ramón, C. Fernández-Cabrera, G. Giménez-Gallego, and A. Romero. 2002. Expression, crystallization and preliminary X-ray diffraction studies on the complete choline-binding domain of the major pneumococcal autolysin. Acta Crystallogr. Sect. D 58:556-558.

9.Ghrayeb, J., H. Kimura, M. Takahara, H. Hsiung, Y. Masui, and M. Inouye. 1984. Secretion cloning vectors in Escherichia coli. EMBO J. 3:2437-2442.

10.Hooper, D.C., J.S. Wolfson, G.L. McHugh, M.D. Swartz, C. Tung, and M.N. Swartz. 1984. Elimination of plasmid pMG110 from Escherichia coli by novobiocin and other inhibitors of DNA gyrase. Antimicrob. Agents Chemother. 25:586-590.

11.Molina, M.A., F.X. Avilés, and E. Querol. 1992. Expression of a synthetic gene encoding potato carboxypeptidase inhibitor using a bacterial secretion vector. Gene 116:129-138.

12.Peränen, J., M. Rikkonen, M. Hyvönen, and L. Kääriäinen. 1996. T7 vectors with a modified T7lac promoter for expression of proteins in Escherichia coli. Anal. Biochem. 236:371373.

13.Petterson, R.F., K. Lundström, J.B. Chattopadhyaya, S. Josephson, L. Philipson, L. Kääriäinen, and I. Palva. 1983. Chemical synthesis and molecular cloning of a STOP oligonucleotide encoding an UGA translation terminator in all three reading frames. Gene 24:15-27.

14.Rico, M., M. Bruix, C. González, R.I. Monsalve, and R. Rodríguez. 1996. ${ }^{1} \mathrm{H}$ NMR assignment and global fold of napin BnIb, a representative $2 \mathrm{~S}$ albumin seed protein. Biochemistry 35:15672-15682.

C.F.-T. and A.R. contributed equally to this work. We are grateful to D. Laurents for critical reading of the manuscript. The first author was supported by a fellowship from the Ministerio de Educación y Ciencia and by a grant from the Residencia de Estudiantes. This work was supported by Inustrial Farmacéutica Cantabria S.A. and by grant nos. PB97-1237 and BIO99-0867 from the Ministerio de Educación y Ciencia (Spain). Address correspondence to Guillermo Giménez-Gallego, Centro de Investigaciones Biológicas - CSIC, Velázquez 144, Madrid, 28006, Spain. e-mail: gimenez gallego@cib.csic.es

Received 15 January 2002; accepted 11 March 2002.

C. Fernández-Tornero, Á. Ramón, M.L. Navarro, J. Varela, and G. Giménez-Gallego Centro de Investigaciones

Biológicas - CSIC

Madrid, Spain

For reprints of this or any other article, contact Reprints@BioTechniques.com 